\title{
Cholangiocarcinoma a rare entity becoming dreadful
}

\author{
S. C. Mohapatra \\ Professor, Dept. of Community Medicine, Dean Academic Affairs \& Coordinator IQAC, SGT University, \\ Gurgaon, Haryana, India
}

Corresponding Author:

Email: vishwamegh@gmail.com

Cholangiocarcinoma (CCCs), also known as bile duct cancer, is a rare form of cancer that is composed of genetically mutated epithelial cells that originate in the bile ducts. The annual incidence rate amounts to 1-2 cases per 100,000 in the Western world ${ }^{1}$ but rates have been rising globally during past few decades. ${ }^{1,2}$ Cholangiocarcinoma is an incurable and lethal cancer unless both the primary tumour and any metastases are fully removed by surgery. No potentially curative treatment exists except surgery, but most people have advanced stage disease at presentation hence become are inoperable cases by the time of diagnosis is arrived at. People with cholangiocarcinoma are generally managed with chemotherapy, radiation therapy, and other palliative care measures even after surgery. It usually ends as "not cured"

An abnormal, liver function tests, jaundice, abdominal pain as well as weight loss, in about $50 \%$ cases, generalized itching (66\%), fever and pale colour of stool and urine are the main signs and symptom of Cholangiocarcinoma, resembling hepatitis with usually elevated bilirubin, alkaline phosphatase and gamma glutamyltransferase levels. However, depending upon the location of the tumour; patients with cholangiocarcinoma with tumours of the bile ducts within the liver have pain without jaundice. Blood tests for liver function in patients might give impression of obstructive jaundice. ${ }^{3,4}$ Primary sclerosing cholangitis (PSC), inflammatory disease and ulcerative colitis(UC) are the main preceding factors although; most people with cholangiocarcinoma have no identifiable risk factors. The incidence of cholangiocarcinoma increases with age, and the disease is slightly more common in men than in women. The prevalence of cholangiocarcinoma in patients with primary sclerosing cholangitis may be as high as $30 \%$, based on autopsy studies.

CCCs are encountered in three anatomic regions: intrahepatic, extrahepatic perihilar, and distal extrahepatic. See the image below.

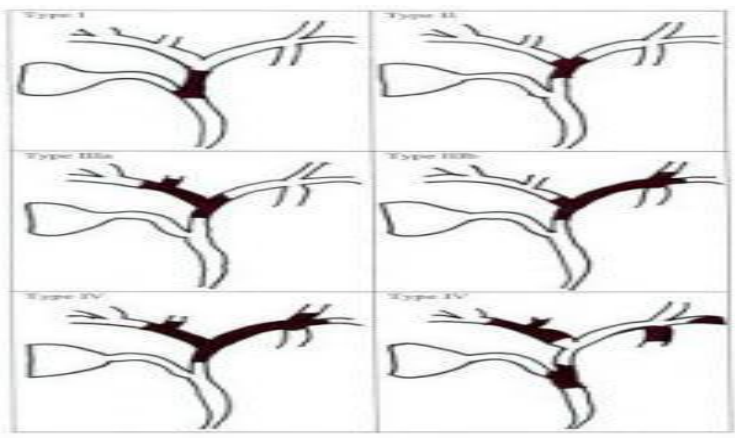

Fig. 1

Epidemiologic studies have suggested that the lifetime risk of developing cholangiocarcinoma for a person with PSC is on the order of $10 \%-15 \%,{ }^{5}$ while autopsy series in the west have shown the rate to be as high as $30 \%$ in this population. ${ }^{6}$ Multiple studies have documented a steady increase in the incidence of intrahepatic cholangiocarcinoma over the past several decades; increases have been seen in North America, Europe, Asia, and Australia. There is a higher prevalence of cholangiocarcinoma in Asia, which has been attributed to endemic chronic parasitic infestation. The occurrence rate of Cholangeo Carcinoma is not known in India. The annual incidence of 1-2 cases per 100,000 people in USA. The liver flukes Opisthor chisviverrini in Thailand, Laos and Vietnam or Clonorchis sinensis in China, Taiwan, eastern Russia, Korea, and Vietnam been associated with cholangiocarcinoma. Control programs aimed at discouraging the consumption of raw and undercooked food have been successful at reducing the incidence of cholangiocarcinoma in some countries. ${ }^{7}$ Patients with chronic liver disease, whether in the form of viral hepatitis B or hepatitis C, alcoholic liver disease, or cirrhosis of the liver due to other causes, are at significantly increased risk of cholangiocarcinoma. HIV infection was also identified in one study as a potential risk factor for cholangiocarcinoma, although it is unclear whether HIV or hepatitis C infections are responsible for the association. ${ }^{7}$ Autopsy series have reported a prevalence of $0.01 \%$ to $0.46 \%$ in the west. Infection with the bacteria Helicobacter bilis and hepaticus species can cause biliary cancer too. Congenital liver abnormalities, such as Caroli's syndrome have been associated with an approximately $15 \%$ lifetime risk of developing cholangiocarcinoma. 
Lynch syndrome II and biliary papillomatosis while gallstones (cholelithiasis) is not found to be associated with cholangiocarcinoma. Prolong exposure to Thorotrast, a radiologic contrast medium of thorium dioxide was calculated towards development of cholangiocarcinoma thus it has been banned in the United States.

Hstopathologically, Cholangiocarcinoma shows features of an adeno-carcinoma of epithelial cells lining of the biliary tract, the actual cell of origin is not known exactly. Cholangiocarcinoma is believed to process by four stages such as early hyperplasia, metaplasia, dysplasia and finally there is development of frank carcinoma.

Diagnosis of CCC is often delayed and at elderly age. There is no specific blood tests either however serum carcinoembryonic antigen (CEA) and CA19-9 are often elevated as a nonspecific evidence, hence cannot be useful as a screening tool for population study. Ultrasound of the liver and biliary tract may be used as helpful particularly in cases with features of obstructive jaundice. Ultrasound can identify obstruction and ductal dilatation and, in some cases, may be sufficient to diagnose cholangiocarcinoma. Computed tomography (CT) scanning may also play an important role in the diagnosis of cholangiocarcinoma. Biopsy through Endoscopic retrograde cholangiopancreatography (ERCP) can be used for diagnosis at the same time putting stents to relieve billiary obstruction. Surgical exploration may be necessary to obtain a suitable biopsy and to accurately stage a patient with cholangiocarcinoma.

Cholangiocarcinoma is conceived as a road to death disease unless the tumour is resected out completely where there is no metastasis. Since the operability of the tumour can only be assessed during surgery in most cases, a majority of patients undergo exploratory surgery unless there is already a clear indication that the tumor is inoperable. There are reports of significant success if early treatment of bile duct cancer with liver transplantation is being received. Adjuvant therapy followed by liver transplantation may have some role.

\section{References}

1. Landis S, Murray T, Bolden S, Wingo P (1998). "Cancer statistics, 1998". CA Cancer J Clin. 48 (1):629. doi:10.3322/canjclin.48.1.6. PMID 9449931.

2. Patel T (2002). "Worldwide trends in mortality from biliary tract malignancies". BMC Cancer. 2:10. doi:10.1186/14712407-2-10. PMC 113759 PMID 11991810

3. Mark Feldman; Lawrence S. Friedman; Lawrence J. Brandt, eds. (21 July 2006). Sleisenger and Fordtran's

Gastrointestinal and Liver Disease (8th ed.). Saunders. pp. 1493-6. ISBN 978-1-4160-0245-1.

4. Tsaitas C, Semertzidou A, Sinakos E (April 2014). "Update on inflammatory bowel disease in patients with primary sclerosing cholangitis". World J Hepatol. 6 (4):178-87.
5. Nagorney D, Donohue J, Farnell M, Schleck C, Ilstrup D (1993). "Outcomes after curative resections of cholangiocarcinoma". Arch Surg. 128 (8):871-7;

6. Nakeeb A, Pitt H, Sohn T, Coleman J, Abrams R, Piantadosi S, Hruban R, Lillemoe K, Yeo C, Cameron J (1996). "Cholangiocarcinoma. A spectrum of intrahepatic, perihilar, and distal tumors". Ann Surg. 224 (4):463-73.

7. Rustagi T, Dasanu CA (2012). "Risk Factors for Gallbladder Cancer and Cholangiocarcinoma: Similarities, Differences and Updates". J Gastrointest Cancer. 43 (2):137-147. 